\title{
Momentum-space study of the effect of bremsstrahlung radiation on the energy of runaway electrons in tokamaks
}

\author{
M. Bakhtiaria) \\ University of Wisconsin-Madison, Madison, Wisconsin 53706 \\ G. J. Kramer \\ Princeton Plasma Physics Laboratory, Princeton, New Jersey 08543 \\ D. G. Whyte \\ University of Wisconsin-Madison, Madison, Wisconsin 53706
}

(Received 30 March 2005; accepted 22 August 2005; published online 5 October 2005)

\begin{abstract}
The dynamics of runaway electrons in tokamak plasmas is studied in momentum space. In this analysis the electrons are accelerated by an electric field while they are decelerated by collisional drag, synchrotron radiation, and bremsstrahlung radiation. It is shown that the inclusion of bremsstrahlung losses into the calculations is important for the interpretation of disruption-mitigation experiments where large amounts of high $Z$ are injected into the plasma. The inclusion of bremsstrahlung reduces the maximum energy that the runaway electrons can gain from the electric field. (C) 2005 American Institute of Physics. [DOI: 10.1063/1.2065368]
\end{abstract}

\section{INTRODUCTION}

In ionized gases with electric fields electrons can be accelerated to very high energies. These electrons are called runaway electrons. ${ }^{1}$ In tokamaks runway electrons are often observed during and after a plasma disruption and during a fast plasma shutdown. ${ }^{2}$ Because of their high energies (up to several $\mathrm{MeV}$ ) these runaway electrons can do significant damage to structures surrounding the plasma. During a plasma disruption the plasma cools down quickly, thereby increasing its resistivity which in turn leads to large electric fields especially when the plasma current is large $(E=\eta j$, with $E$ being the electric field, $\eta$ being the resistivity, and $j$ being the current density). Runaway electrons are generated when the electric force acting on the electrons exceeds the friction force between electrons and plasma particles so that they can accelerate freely to high energies. The maximum energy that a runaway electron can reach, however, is limited through various mechanisms. Different energy limits for runaway electrons in tokamaks have been studied ${ }^{3,4}$ and the most attractive mechanism for limiting the energy of runaway electrons in tokamaks is synchrotron radiation which is generated when a relativistic electron moves on a curved path. ${ }^{5}$ A second mechanism that can limit the energy of runaway electrons is bremsstrahlung which is generated when electrons are deflected by ions. Although bremsstrahlung has been used widely to diagnose runaway electrons it has not been considered to limit the maximum energy that the runaway electrons can gain. For hydrogen plasmas with low effective charges $\left(Z_{\text {eff }}\right)$ this is true, but recently there has been an interest to use high- $Z$ impurity injection for mitigating runaway electron currents during and after disruptions. In these experiments massive amounts of high- $Z$ impurities are injected into the plasma for a fast plasma shutdown by means of a radiation collapse. Because of the injection of

${ }^{a)}$ Electronic mail: bakhtiar@engr.wisc.edu massive amounts of high- $Z$ impurities, $Z_{\text {eff }}$ increases significantly and bremsstrahlung cannot be ignored anymore as a limiting factor for the energy of runaway electrons. ${ }^{8}$

In this paper we study the effects of bremsstrahlung radiation together with synchrotron radiation and collisions on runaway electrons using a test particle description and we consider their orbits in momentum space.

In earlier studies of runaway electrons in momentum space, synchrotron radiation and collisions were only taken into account. ${ }^{9-11}$ The electron trajectory in momentum space is developed in Sec. II while in Sec. III the effects of inclusion of bremsstrahlung on the maximum energy that runaway electrons can gain is calculated. This is followed by a discussion of the combined effects of synchrotron radiation and bremsstrahlung for current and future machines (Sec. IV) and conclusions are drawn in Sec. V.

\section{ELECTRON TRAJECTORY IN MOMENTUM SPACE}

Relativistic electrons in a tokamak plasma are subject to an acceleration force due to the parallel electric field $E_{\|}$and various decelerations forces. Deceleration is caused by collisions and radiation losses. In the following we investigate the role of bremsstrahlung radiation on runaway electrons that are generated during plasma shutdown scenarios with large high- $Z$ impurity gas injections.

We can write the parallel and total force balance for relativistic electrons as follows where we have included the acceleration force due to $E_{\|}$and deceleration forces due to collisions and radiation: ${ }^{12}$

$$
\frac{d p_{\|}}{d t}=e E_{\|}-\frac{n_{e} e^{4} \ln \Lambda m_{e}}{4 \pi \epsilon_{0}^{2}} \gamma\left(Z_{\mathrm{eff}}+1+\gamma\right) \frac{p_{\|}}{p^{3}}-\left(F_{S}+F_{B}\right) \frac{p_{\|}}{p},
$$




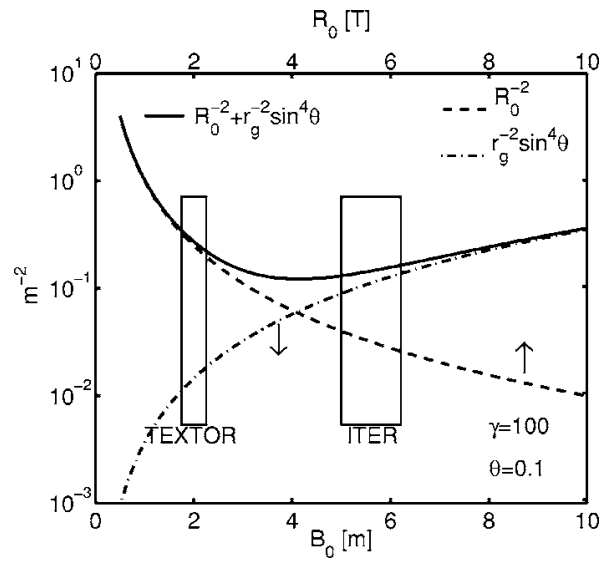

FIG. 1. Contributions to synchrotron radiation of the guiding center motion $\left(R_{0}^{-2}\right)$ as a function of major radius $R_{0}$, and gyromotion $\left(\sin ^{4} \theta / r_{g}^{2}\right)$ as a function of magnetic field $B_{0}$.

$$
\frac{d p}{d t}=e E_{\|} \frac{p_{\|}}{p}-\frac{n_{e} e^{4} \ln \Lambda m_{e}}{4 \pi \epsilon_{0}^{2}} \frac{\gamma^{2}}{p^{2}}-\left(F_{S}+F_{B}\right),
$$

where $p_{\|}$is the electron momentum in toroidal direction, $p$ is the total electron momentum, $\gamma$ is the relativistic factor, $e$ is electron charge, $m_{e}$ is electron mass, $n_{e}$ the electron density, $E_{\|}$is parallel electric field, $Z_{\mathrm{eff}}$ is the effective ionic charge, $\ln \Lambda$ is the coulomb logarithm, and $F_{S}$ and $F_{B}$ are the synchrotron radiation and bremsstralung drag forces, respectively. The first term in the right-hand side of Eqs. (1) and (2) is the acceleration force and the second term is the deceleration force caused by collisions. The synchrotron radiation drag force $F_{S}$ is given by ${ }^{12}$

$$
F_{S}=\frac{2}{3} r_{e} m_{e} c^{2}\left(\frac{v}{c}\right)^{3} \gamma^{4}\left\langle\frac{1}{R^{2}}\right\rangle
$$

with average radius of curvature over one gyrorotation,

$$
\left\langle\frac{1}{R^{2}}\right\rangle \simeq \frac{1}{R_{0}^{2}}+\frac{\sin ^{4} \theta}{r_{g}^{2}}
$$

with $R_{0}$ being the tokamak plasma major radius, $\theta$ the pitch angle, $r_{g}=p_{\perp} /(e B)$ the relativistic electron gyroradius with $p_{\perp}$ being the perpendicular momentum, $r_{e}=e^{2} /\left(4 \pi \epsilon_{0} m_{e} c^{2}\right)$ the classical electron radius, $v$ electron velocity, and $v / c$ $=\left(\gamma^{2}-1\right)^{1 / 2} / \gamma$.

The first term on the right-hand side of Eq. (4) is determined mainly by the motion of the particle along the field lines (guiding center motion) while the second term is the contribution from the gyromotion around the field line. Both contributing parts are shown in Fig. 1 as well as sum of them for an electron with $\gamma=100$ and $\theta=0.1$. The contribution from the guiding center $\left(R_{0}^{-2}\right)$ decreases with increasing major radius $R_{0}$. The contribution from the gyromotion increases with increasing magnetic field $B$. The sum of these components for a large machine is almost the same as that for a small machine.

The bremsstrahlung friction force can be written as ${ }^{13}$

$$
F_{B}=\frac{4}{137} n_{e}\left(Z_{\mathrm{eff}}+1\right) m_{e} c^{2} \gamma r_{e}^{2}\left(\ln 2 \gamma-\frac{1}{3}\right),
$$

for a one-species plasma with an ion density of $n_{i}$ and atomic number of $Z=Z_{\text {eff }}$ and electron density of $n_{e}=n_{i} Z_{\text {eff }}$. Note that the synchrotron radiation depends on the toroidal magnetic field, the major radius of the tokamak, and electron velocity, while the bremsstrahlung drag force is independent of the magnetic field and major radius, and can be increased by increasing the impurity concentration in the plasma. In normal fusion tokamak operations $Z_{\text {eff }}$ is low, just above one, but in case of an emergency when the tokamak has to be shutdown fast, a massive amount of high- $Z$ impurities can be injected raising $Z_{\text {eff }}$ to a value close to $Z_{\text {eff }}=Z_{\text {imp }}$.

Solving Eqs. (1) and (2) gives the test particle (runaway electron) trajectory in momentum space. It is more convenient to use normalized variables to save calculation time and to simplify the calculations. Here we use the following new variables: $q=p / m_{e} c$ as normalized momentum, $\tau=\nu t$ is the time multiplied by collision frequency with $\nu$ $=n_{e} e^{4} \ln \Lambda /\left(4 \pi \epsilon_{0}^{2} m_{e}^{2} c^{3}\right)$, and $D=E_{\|} / E_{c}$ as normalized electric field with $E_{c}=n_{e} e^{3} \ln \Lambda /\left(4 \pi \epsilon_{0}^{2} m_{e} c^{2}\right)$ the critical electric field for runaway electron generation. Now the normalized forms of Eqs. (1) and (2) become:

$$
\begin{aligned}
\frac{d q_{\|}}{d \tau}= & D-\gamma(\alpha+\gamma) \frac{q_{\|}}{q^{3}}-\left(F_{\mathrm{gc}}+F_{\mathrm{gy}} \frac{q_{\perp}^{2}}{q^{4}}\right) \gamma^{4}\left(\frac{v}{c}\right)^{3} \frac{q_{\|}}{q} \\
& -F_{\mathrm{br}} \alpha \gamma\left(\ln 2 \gamma-\frac{1}{3}\right) \frac{q_{\|}}{q}, \\
\frac{d q}{d \tau}= & D \frac{q_{\|}}{q}-\frac{\gamma^{2}}{q^{2}}-\left(F_{\mathrm{gc}}+F_{\mathrm{gy}} \frac{q_{\perp}^{2}}{q^{4}}\right) \gamma^{4}\left(\frac{v}{c}\right)^{3} \\
& -F_{\mathrm{br}} \alpha \gamma\left(\ln 2 \gamma-\frac{1}{3}\right),
\end{aligned}
$$

where $\quad \alpha=1+Z_{\text {eff }}, \quad F_{\text {gc }}=F_{\text {gy }}\left[m_{e} c /\left(e B_{0} R_{0}\right)\right]^{2}, \quad F_{\text {gy }}$ $=2 \epsilon_{0} B_{0}^{2} /\left(3 n_{e} \ln \Lambda m_{e}\right), B_{0}$ is toroidal magnetic field, and $F_{\mathrm{br}}$ $=(137 \pi \ln \Lambda)^{-1}$. Here, $F_{\mathrm{gc}}$ and $F_{\mathrm{gy}}$ describe the contribution of guiding center motion and electron gyromotion, respectively, to the synchrotron radiation.

Instead of using the total and parallel momenta in our considerations, it is easier to use the parallel and perpendicular momenta $\left(q_{\|}, q_{\perp}\right)$. From $q_{\|}$and $q_{\perp}$ we can then calculate easily the critical electric fields, pitch angles, and energy limits for runaway electrons. The perpendicular momentum $q_{\perp}$ is obtained from Eqs. (6), (7), and $q^{2}=q_{\|}^{2}+q_{\perp}^{2}$ and is given by

$$
\begin{aligned}
q_{\perp} \frac{d q_{\perp}}{d \tau}= & \frac{1}{q}\left[\gamma(\alpha+\gamma) \frac{q_{\|}^{2}}{q^{2}}-\gamma^{2}\right. \\
& -\left(F_{\mathrm{gc}}+F_{\mathrm{gg}} \frac{q_{\perp}^{2}}{q^{4}}\right) \gamma^{4}\left(\frac{v}{c}\right) q_{\perp}^{3} \\
& \left.-F_{\mathrm{br}} \alpha \gamma\left(\ln 2 \gamma-\frac{1}{3}\right) q_{\perp}^{2}\right] .
\end{aligned}
$$

Solving Eqs. (6) and (8) gives the trajectories of runaway electrons in momentum or $\left(q_{\|}, q_{\perp}\right)$ space. An example is 

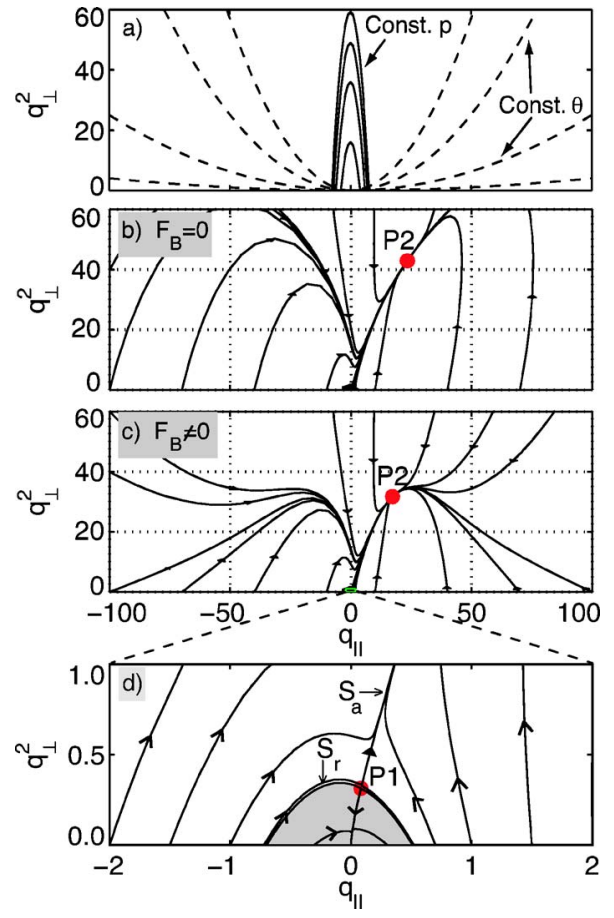

FIG. 2. (a) Constant total momentum $p$ and pitch angle $\theta$ surfaces in momentum space. The trajectories of runaway electrons in momentum space (b) taking into account the effect of synchrotron radiation only, (c) taking into account both synchrotron radiation and bremsstrahlung, and (d) a close up of the plot in the low momentum range which is identical for both cases. Electrons moving in the shaded area return to the thermal population and electrons outside of the shaded area are runaway electrons. The plasma parameters are $D=30, \alpha=55\left(Z_{\mathrm{eff}}=54\right), F_{\mathrm{gy}}=0.65$, and $F_{\mathrm{gc}}=2.3 \times 10^{-7}$.

given in Fig. 2. In Fig. 2 several constant momentum and pitch angle surfaces are shown in the momentum space. We have solved the equations for two cases: (i) taking into account the synchrotron radiation only [Fig. 2(b)] and (ii) taking into account the synchrotron radiation and the bremsstrahlung [Fig. 2(c)]. From this figure it can be seen that the high momentum range is modified strongly by the inclusion of bremsstrahlung while the low momentum range [Fig. 2(d)] is not affected by bremsstrahlung. The effects of bremsstrahlung only show up at high momenta. The parameters that were used for this figure are $D=30, \alpha=55\left(Z_{\text {eff }}\right.$ $=54$ for a xenon plasma), $F_{\mathrm{gy}}=0.65$, and $F_{\mathrm{gc}}=2.3 \times 10^{-7}$. Depending on the initial momentum and pitch angle, electrons have different traces in momentum space. A separatrix separates the trajectories of electrons with different momenta. Those with momenta larger than the separatrix $S_{r}$ [above the shaded area in Fig. 2(d)] are runaway electrons and accelerate (or decelerate) to a stable point, $P_{2}$ in Figs. 2(b) and 2(c), located on a second separatrix $S_{a}$. Electrons with less momentum (i.e., inside the separatrix $S_{r}$ ) are slowed down and return to the bulk plasma population [shaded area in Fig. 2(d)]. One can see clearly from Figs. 2(b) and 2(c) that bremsstrahlung significantly affects the trajectories of runaway electrons in the momentum space. The position of the stable point $P_{2}$ shifts also to a lower-energy when bremsstrahlung is taken into account. In fact high-energy electrons are continuously decelerating toward point $P_{2}$ when brems- strahlung is considered (see, for example, the trace of an electron with initial $q_{\|}=100$ and $q_{\perp}=0$.)

In the case of synchrotron radiation only [Fig. 2(b)] the runaway electrons have to move to the high pitch angle region (or obtain a high $q_{\perp}$ ) where the synchrotron radiation is strong before they slow down to the stable point $P_{2}$. When bremsstrahlung radiation is included, the runaway electrons do not need to reach very high pitch angles before they can reach $P_{2}$ so the inclusion of bremsstrahlung is important for the modeling of runaway electrons.

In momentum space there are two stable points, $P_{1}$ at the crossing of separatices $S_{r}$ and $S_{a}$ and $P_{2}$ on $S_{a}(2 d)$. Electrons moving on the separatrix $S_{r}$ are finally stopped (de)accelerating at the saddle point $P_{1}$ and return to the bulk plasma with almost zero momentum. Similarly, runaway electrons stop (de)accelerating at the stable point $P_{2}$. Saddle point $P_{1}$ is determined by the collisions while stable point $P_{2}$ is determined by radiation. ${ }^{12}$ The stable point $P_{2}$ in our calculation has shifted to a lower momentum region due to the effect of bremsstrahlung. More details on the behavior of separatrices can be found in Refs. 9, 10, and 12 .

For the determination of energy limits for runaway electrons the stable points are very important. At those points the net force working on the electrons is zero which means that $\dot{q}=0$ (or $\dot{q}_{\|}=0$ and $\dot{q}_{\perp}=0$ ) ( $\dot{f}$ is the derivative of $f$ with respect to time). This leads to analytical expressions for the electric field $D$ and the pitch angle $\theta_{s}$ at $P_{1}$ and $P_{2}$. Considering $\dot{q}=0$ we obtain

$$
\begin{aligned}
D= & \frac{\gamma_{s}^{2}}{\cos \theta_{s}\left(\gamma_{s}^{2}-1\right)}\left[1+F_{\mathrm{gy}} \frac{\left(\gamma_{s}^{2}-1\right)^{3 / 2}}{\gamma_{s}} \sin ^{2} \theta_{s}\right. \\
& \left.+F_{\mathrm{gcbr}} \frac{\left(\gamma_{s}^{2}-1\right)^{5 / 2}}{\gamma_{s}}\right],
\end{aligned}
$$

and $\dot{q}_{\perp}$ gives the expression for the pitch angle,

$$
\begin{aligned}
\cos ^{2} \theta_{s}= & 1+\frac{F_{\mathrm{gcbr}}\left(\gamma_{s}^{2}-1\right)}{2 F_{\mathrm{gy}}}+\frac{\alpha+\gamma_{s}}{2 F_{\mathrm{gy}}\left(\gamma_{s}^{2}-1\right)^{3 / 2}} \\
& \times\left(1-\left\{\frac{4 \alpha F_{\mathrm{gy}}\left(\gamma_{s}^{2}-1\right)^{3 / 2}}{\left(\alpha+\gamma_{s}\right)^{2}}\right.\right. \\
& \left.\left.+\left[1+\frac{F_{\mathrm{gc}}\left(\gamma_{s}^{2}-1\right)^{5 / 2}}{\alpha+\gamma_{s}}\right]^{2}\right\}^{1 / 2}\right),
\end{aligned}
$$

where $F_{\mathrm{gcbr}}=F_{\mathrm{gc}}+F_{\mathrm{br}} \alpha \gamma(\ln 2 \gamma-1 / 3)\left(\gamma^{2}-1\right)^{-3 / 2}$ and $\gamma_{s}$ is the relativistic factor at the stable point under consideration $\left(P_{1}\right.$ or $P_{2}$ ).

Plots of $\gamma_{s}$ versus the normalized electric field $D$ are shown in Fig. 3 for $\alpha=4\left(Z_{\text {eff }}=3\right.$ and $\alpha=55\left(Z_{\text {eff }}=54\right)$. Three cases were calculated with (i) no limiting radiation, (ii) only synchrotron radiation, and (iii) both synchrotron radiation and bremsstrahlung taken into account. In the two cases with radiation taken into account there is a clear threshold value for $D\left(D_{\text {crit }}\right)$ which corresponds to the critical electric field above which runaway electrons can be generated. Above $D_{\text {crit }}$ a constant $D$ crosses each curve at two points. The first crossing at low $\gamma_{s}$ corresponds to saddle point $P_{1}$ and is determined by the collisional drag force while the second crossing at high $\gamma_{s}$ corresponds to the stable point $P_{2}$ which 

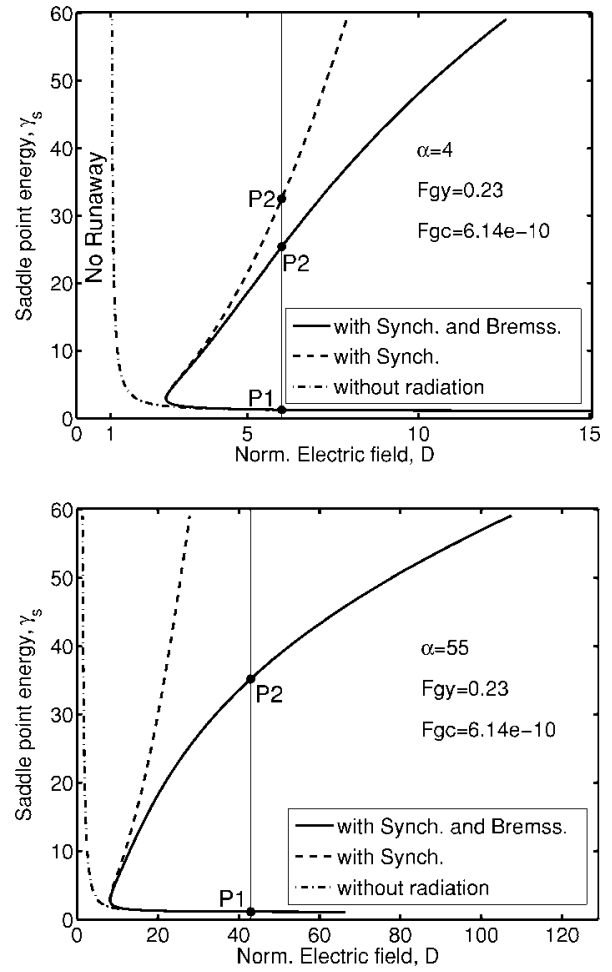

FIG. 3. The normalized electric field vs energy at singular points $P_{1}$ and $P_{2}$ for $Z_{\text {eff }}=3$ (top) and $Z_{\text {eff }}=54$ (bottom). The ITER parameters were used $\left(F_{\mathrm{gy}}=0.23\right.$ and $\left.F_{\mathrm{gc}}=6.14 \times 10^{-10}\right)$.

is determined by radiation losses. The high $\gamma_{s}$ crossing is the high energy limit for runaway electrons. From Fig. 3 one may find that $D_{\text {crit }}$ is higher for larger $\alpha$ (or higher $Z_{\text {eff }}$ ) mainly due to the collisional drag force and synchrotron radiation. For $D$ just above $D_{\text {crit }}$ bremsstrahlung has no effect but the effect of bremsstrahlung appears when $D$ is well above $D_{\text {crit }}$.

\section{BREMSSTRAHLUNG ENERGY LIMIT}

The stable point $P_{2}$ determines the maximum energy or energy limit that runaway electrons can obtain in a tokamak and it is obtained from Eqs. (9) and (10). This energy limit is determined by radiation losses. It is shown as a function of the normalized electric field $D$ in Fig. 3 for $\alpha=4\left(Z_{\text {eff }}=3\right)$ and $\alpha=55\left(Z_{\text {eff }}=54\right)$. From this figure it can be seen that the maximum energy gain decreases significantly when bremsstralung is included in the calculations together with synchrotron radiation compared to the synchrotron radiation only case, especially for higher $D$ 's.

The effect of increasing the effective charge is twofold: it increases the critical energy above which runaway electrons are generated and it decreases the maximum energy that runaway electrons can reach. Both effects are very favorable to limit possible damage that runaway electrons can do to structures surrounding the plasma in fusion machines.

\section{DISCUSSION}

From Eq. (3) one can see that the synchrotron radiation is dependent on the machine size in a way that its importance decreases for tokamaks with large major radii $R_{0}$. For a large

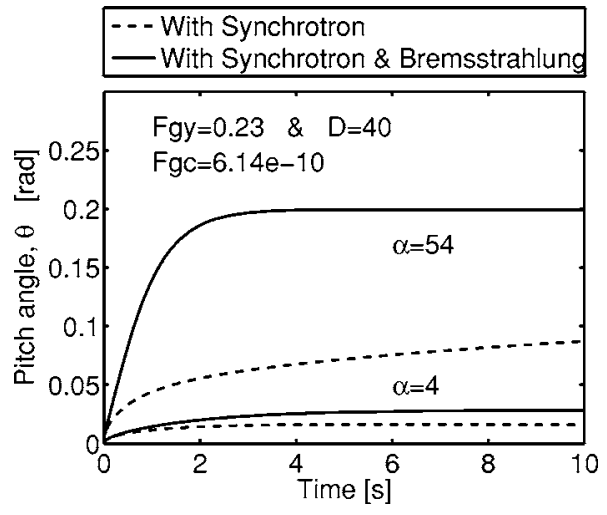

FIG. 4. Change in the pitch angle for a runaway electron with an energy of $\gamma=200(W=100 \mathrm{MeV})$ in an ITER plasma with different effective charges $(\alpha)$. The change in the pitch angle is strong when the effective charge is high. This leads to a strong synchrotron radiation. Taking into account the effect of bremsstrahlung enhances the change in the pitch angle which leads to a stronger synchrotron radiation even for plasmas with low effective charges.

tokamak such as International Thermonuclear Experimental Reactor (ITER) with $R_{0}=8 \mathrm{~m}$ synchrotron radiation gets rather weak. In fact synchrotron radiation depends on the magnetic field and major radius of the device which are difficult to change in order to enhance the synchrotron radiation and mitigate the deleterious effects of runaway electrons. For bremsstrahlung radiation the situation is different. It is independent of the machine size and machine parameters such as $R_{0}$ and $B_{T}$. Bremsstrahlung radiation increases as a function of $Z_{\text {eff }}$ and runaway electron energy. Increasing the effective charge increases the pitch angle of high-energy electrons which in turn leads to an enhancement of the synchrotron radiation. The influence of the effective charge on synchrotron radiation is shown in Fig. 4 which shows the evolution of the pitch angle for a $100 \mathrm{MeV}$ electron moving initially in a parallel direction (zero pitch angle). As shown in Fig. 4 the change in the pitch angle is strong with high $Z$ leading to stronger synchrotron radiation. The change in the pitch angle is stronger when bremsstrahlung is taken into account.

Therefore, increasing the effective charge of the plasma by injecting heavy impurities can be a feasible method to mitigate the effects of disruption-generated runaway electrons in tokamaks. ${ }^{8}$

We have used a test particle approach as was formulated in Ref. 9 for calculating the effects of bremsstrahlung on the high energy limit of runaway electrons. In this formulation, individual electrons are followed in phase space while the electron-electron and electron-ion scatterings are taken into account by averaging over the stopping power and pitch angle scattering. In a Fokker-Plank approach the electrons do not follow the orbit exactly as calculated in the test particle approach in a process called path broadening. ${ }^{9}$ A consequence of path broadening is that one must take care in calculating averaged quantities from the Fokker-Plank equation, and the averaging procedure may have some effect on the exact location of the stationary points. However, because the path broadening decreases with increasing energy ${ }^{9}$ it is expected that the high energy limit for runaway electrons is 
only slightly affected by using a Fokker-Plank approach instead. This expectation is supported by the fact that a comparison between a momentum-space description of highenergy avalanche of runaway electrons in tokamaks between a Fokker-Planck solution and a Monte Carlo simulation produce very similar results. ${ }^{11,14}$

\section{CONCLUSIONS}

The dynamics of runaway electrons in momentum space was studied whereby not only synchrotron radiation but also bremsstrahlung was considered as a mechanism to limit the energy of runaway electrons in tokamaks. The supression of runaway electrons during fast plasma shutdown scenarios is of paramount importance in order to avoid damage to plasma-facing structures. It was found that bremsstrahlung can greatly reduce the maximum energy that runaway electrons can gain in high electric fields. The effect of bremsstrahlung increases with the effective charge of plasma and the energy of electrons.

The effective charge and electrical field set an energy limit for runaway electrons. At this energy limit the energy gain from the electrical field is compensated by bremsstralung and synchrotron radiation losses.

The maximum energy gain was reduced by $40 \%$ when bremsstrahlung was taken into account compared to the case in which only synchrotron radiation was taken into account. The bremsstrahlung and synchrotron radiation, which limit the energy of runaway electrons, can be increased by increasing the effective charge of plasma. An enhancement of the effective charge of the plasma also increases the critical electric field above which runaway electrons are generated.

The bremsstrahlung radiation increases with $Z_{\text {eff }}^{2}$ and is independent of the machine size while synchrotron radiation increases with $Z_{\mathrm{eff}}$ and decreases with the size of device. This makes bremsstrahlung a reliable way of reducing the number and maximum energy of runaway electrons during disruption-mitigation operation with a massive impurity injection.

\section{ACKNOWLEDGMENTS}

This work was supported by the US Department of Energy under Grant No. DE-FG02-04ER54762 and by Japan Atomic Energy Research Institute during the author's (M.B.) research period in Naka Fusion Research Establishment.

${ }^{1}$ H. Dreicer, Phys. Rev. 115, 238 (1959).

${ }^{2}$ R. Yoshino, T. Kondoh, Y. Neyatani, K. Itami, Y. Kawano, and N. Isei, Plasma Phys. Controlled Fusion 39, 313 (1997).

${ }^{3}$ H. Knoepfel and D. A. Spong, Nucl. Fusion 19, 785 (1979).

${ }^{4}$ J. R. Martin-Solis, B. Esposito, R. Sanchez, and J. D. Alvarez, Phys. Plasmas 6, 238 (1999).

${ }^{5}$ F. Andersson, P. Helander, and L.-G. Eriksson, Phys. Plasmas 8, 5221 (2001).

${ }^{6}$ D. G. Whyte, T. C. Jernigan, D. A. Humphreys, A. W. Hyatt, C. J. Lasnier, P. B. Parks, T. E. Evans, M. N. Rosenbluth, P. L. Taylor, A. G. Kellman et al., Phys. Rev. Lett. 89, 055001 (2002).

${ }^{7}$ M. Bakhtiari, Y. Kawano, H. Tamai, Y. Miura, R. Yoshino, and Y. Nishida, Nucl. Fusion 42, 1197 (2002).

${ }^{8}$ M. Bakhtiari, G. J. Kramer, M. Takechi, H. Tamai, Y. Miura, Y. Kusama, and Y. Kamada, Phys. Rev. Lett. 94, 215003 (2005).

${ }^{9}$ G. Fussmann, Nucl. Fusion 19, 327 (1979).

${ }^{10}$ V. Fuchs, R. A. Cairns, C. N. Lashmore-Davies, and M. M. Shoucri, Phys. Fluids 29, 2931 (1986).

${ }^{11}$ P. B. Parks, M. N. Rosenbluth, and S. V. Putvinski, Phys. Plasmas 6, 2523 (1999).

${ }^{12}$ J. R. Martin-Solis, J. D. Alvarez, R. Sanchez, and B. Esposito, Phys. Plasmas 5, 2370 (1998)

${ }^{13}$ D. J. Jackson, Classical Electrodynamics, 3rd ed. (Wiley, New York, 1999).

${ }^{14}$ M. Rosenbluth and S. Putvinski, Nucl. Fusion 37, 1355 (1997). 